\title{
EchoGéo
}

$33 \mid 2015$

Activisme, participation, contestation : la place des habitants dans les processus de patrimonialisation en périphéries urbaines

\section{Itinéraires et patrimoines urbains}

\author{
Jean-Louis Chaléard
}

\section{OpenEdition}

\section{Journals}

Electronic version

URL: https://journals.openedition.org/echogeo/14362

DOI: 10.4000/echogeo.14362

ISSN: 1963-1197

Publisher

Pôle de recherche pour l'organisation et la diffusion de l'information géographique (CNRS UMR 8586)

Electronic reference

Jean-Louis Chaléard, "Itinéraires et patrimoines urbains", EchoGéo [Online], 33 | 2015, Online since 30

September 2015, connection on 11 August 2021. URL: http://journals.openedition.org/echogeo/14362 ; DOI: https://doi.org/10.4000/echogeo.14362

This text was automatically generated on 11 August 2021.

EchoGéo est mis à disposition selon les termes de la licence Creative Commons Attribution - Pas d'Utilisation Commerciale - Pas de Modification 4.0 International (CC BY-NC-ND) 


\title{
Itinéraires et patrimoines urbains
}

\author{
Jean-Louis Chaléard
}

1 Le patrimoine a pris une dimension importante dans nos sociétés et a vu son contenu s'élargir depuis quelques décennies. Le présent numéro d' EchoGéo s'intéresse aux formes récentes de patrimonialisation dans les banlieues et les anciennes cités ouvrières en France, qui conduisent aussi à de nouvelles pratiques de la ville. Au-delà, ces évolutions posent des questions plus générales quant aux dynamiques urbaines, au déclin et à la requalification de certains quartiers de villes en France et dans les pays anciennement industrialisés. Dans ce contexte de patrimonialisation et de mutations urbaines, une large place est faite aux citadins, résidents des quartiers concernés et acteurs de ces dynamiques.

2 EchoGéo focalise souvent ses thèmes sur des terres lointaines, plus que sur l'hexagone. Cette fois, le dossier de la rubrique Sur le champ dirigé par E. Auclair et A. Herzog, consacré à la place des habitants dans les processus de patrimonialisation dans les banlieues, est centré sur la France. Les cas analysés sont néanmoins variés et permettent la mise en lumière de facettes multiples du sujet abordé. Deux études sont situées dans la communauté d'agglomérations de Plaine Commune au nord de Paris, dans l'ancienne «banlieue rouge ». Dans la première, S. Jacquot s'interroge sur la place que confèrent les politiques patrimoniales aux habitants. Dans la seconde, G. DjamentTran examine quand et à quelles conditions la patrimonialisation du logement social aboutit et quel rôle y jouent les habitants. Les trois autres textes portent sur des cités et des grands ensembles, aux histoires et aux conceptions différentes, localisés dans des villes d'importance inégale. A. Chenevez s'intéresse au Musée Urbain Tony Garnier dans la métropole lyonnaise, dont l'exemple souligne les difficultés et les enjeux de formes récentes de patrimonialisation. $R$. Kaddour s'interroge sur la prise en compte de la pluralité des mémoires d'habitants dans de grands ensembles à la périphérie sud-est de Saint-Étienne. Enfin, D. Miralles Buil traite du rôle des politiques municipales dans la mise en patrimoine à partir de l'exemple du quartier de l'Arsenal, à cheval sur les communes de Roanne, ville moyenne de la Loire, et Mably, située à sa périphérie. Les exemples retenus portent sur des espaces à fort passé industriel et populaire : des cités ouvrières et des cités jardins de la première moitié du $\mathrm{XX}^{\mathrm{e}}$ siècle aux grands ensembles de la seconde moitié, édifiés en général en banlieue. Ils ont connu de profonds 
bouleversements liés aux effets de la désindustrialisation et aux politiques d'aménagement urbain, ainsi qu'aux évolutions sociales (que ce soit une gentrification ou une paupérisation).

3 Les textes soulignent tous l'évolution de la notion de patrimoine. S. Jacquot distingue ainsi le nouveau patrimoine de Plaine Commune, centré sur les habitants et l'habitat populaire, d'une conception plus ancienne et plus « classique » portée notamment par le syndicat d'initiative de Saint-Denis, autour de la basilique. Il peut s'agir d'un patrimoine architectural mais aussi de l'histoire d'une cité, comme l'étudie D. Miralles Buil dans le quartier de l'Arsenal à Roanne et Mably. Pour A. Chenevez, le Musée Urbain Tony Garnier à Lyon fait partie de ces «nouvelles causes du patrimoine » soutenues, de façon significative, par des acteurs extérieurs aux institutions culturelles (préfet de département, sociologues, etc.). Cela suppose de nouveaux arguments pour accéder à cette patrimonialisation, et l'implication des habitants. C'est aussi pour cette raison que les articles se devaient de s'interroger sur la place des résidents dans ces évolutions.

4 Tous les textes font valoir aussi que la mise en patrimoine, processus complexe et multidimensionnel (culturel, politique, social, spatial), est le fait d'acteurs variés : État, municipalités, sociétés HLM, associations. Les motivations en sont diverses : se côtoient des logiques d'aménagement et de requalification urbains, de marketing territorial, de mobilisation des habitants, de sauvegarde d'une mémoire des lieux... Le rôle des élus est fondamental. Cela se vérifie à Plaine Commune mais aussi dans la cité de l'Arsenal où la patrimonialisation est portée par la municipalité du côté de Mably alors que la ville de Roanne s'en désintéresse. Le contexte national et international actuel (songeons aux déclarations de l'UNESCO) est marqué par des injonctions à la participation des habitants aux politiques patrimoniales. Mais ces derniers constituent eux-mêmes des catégories diverses par leur ancrage territorial, leur origine sociale. Les arguments de justice patrimoniale, invoqués dans l'action des résidents et des édiles, sont surtout portés par classes moyennes cultivées, comme dans la cité Tony Garnier. G. Djament-Tran souligne l'implication souvent faible des habitants des logements sociaux dans les quartiers populaires de Plaine Commune. À Saint-Étienne, dans les cas étudiés, la patrimonialisation fait une place inégale aux différentes mémoires des habitants: celles des plus anciens locataires, ouvriers et classes moyennes, sont valorisées, alors que celles des habitants plus précaires sont délaissées.

5 La mise en valeur de ces patrimoines est souvent l'occasion de formes nouvelles de tourisme: visites de découverte, participation à des manifestations festives de résidents, hébergement à bas coût, etc. L'interview d'É. Bierry, responsable de l'association Promenades Urbaines, par L. Boukhris, dans la rubrique Sur le métier, prolonge le dossier Sur le champ, dans la mesure où cette association organise des parcours pour découvrir l'architecture, les formes d'urbanisme, les paysages et jusqu'à la vie des habitants de Paris ou de sa banlieue. Elle réunit des promeneurs venant de tous les horizons. Ces actions contribuent à la valorisation du patrimoine urbain, au sens large. L'interview est aussi l'occasion de montrer l'intérêt de l'étude des paysages et de la démarche des géographes, qui débouchent sur une approche renouvelée des territoires, dont É. Bierry fournit quelques exemples.

6 La question de la patrimonialisation s'inscrit dans une réflexion sur l'habiter urbain et l'appropriation collective et inégalitaire des villes. En ce sens, la rubrique Sur l'écrit enrichit également le thème central du présent numéro d'EchoGéo en s'intéressant aux relations entre commerce de détail et changement social urbain, à travers la 
présentation de dix articles retenus parmi une abondante littérature sur la géographie du commerce de détail. L'intérêt de la présentation est de croiser l'évolution des quartiers et l'évolution de l'activité marchande, ce qui est rarement fait. Les exemples analysés, en Europe, en Amérique du Nord, en Australie, offrent un éventail riche à la fois de l'évolution du commerce comme secteur économique et des commerçants comme acteurs. Sont présentés des cas de déclin du commerce, lié à des changements de clientèle ou au dépeuplement, et de dynamisme lors de processus de requalification que les commerçants peuvent accompagner.

7 Le dernier article de la rubrique Sur le vif de C. Vandermotten et P. Medina Lockhart porte sur les élections régionales en Europe. On n'y trouve que de lointains échos de l'évolution économique et sociale que décrivent les textes sur la patrimonialisation. Il montre que les cartes des résultats électoraux doivent avant tout être interprétées dans les contextes nationaux, soulignant la faible adhésion aux formes dominantes de la construction européenne, phénomène particulièrement sensible en Grande-Bretagne et dans les nouveaux États membres. L'analyse dans cet article se situe à une échelle totalement différente de celle des textes sur le patrimoine, attachés au local. Il illustre la diversité de la démarche géographique autour des territoires. 